\title{
Stairlike fracture of the sternum
}

\author{
Recep Sade MD, Yener Aydin MD
}

— Cite as: CMAJ 2017 July 10;189:E914. doi: 10.1503/cmaj.161344

A 30-year-old man, who had been involved in a traffic collision four hours previously, was transferred to our university hospital from a rural hospital. He was not wearing a seat belt while driving and had an isolated sternal fracture with severe displacement after hitting the steering wheel (Figure 1).

Examination using contrast-enhanced computed tomography showed no evidence of cardiac or vascular injury. The patient had normal serum troponin levels, and normal electrocardiogram and echocardiograph results. Because of his severe chest pain, he was admitted to hospital for pain control and monitoring. We offered sternal fixation; however, he declined surgery and was discharged on the third day.

Sternal fracture caused by traffic collisions may occur in isolation or with fractures of the ribs, clavicle or spine. It has an incidence of $0.64 \%$ to $4.8 \%$ in thoracic trauma. ${ }^{1}$ In severe trauma, it may be associated with cardiac contusion, vascular injuries, lung contusion and hemopneumothorax. Although sternal fractures are usually treated with pain control alone, indications for surgical intervention include severe displacement, severe or persistent pain, nonunion, respiratory failure or dependency on mechanical ventilation and restricted movement of the trunk. ${ }^{1}$

The prognosis for isolated sternal fractures is excellent. Death usually occurs in patients with polytrauma, with mortality from $25 \%$ to $45 \% .^{2,3}$ In the absence of other injuries or severe pain, patients with isolated sternal fractures do not need admission to hospital.

\section{References}

1. Knobloch K, Wagner S, Haasper C, et al. Sternal fractures occur most often in old cars to seat-belted drivers without any airbag often with concomitant spinal injuries: clinical findings and technical collision variables among 42,055 crash victims. Ann Thorac Surg 2006;82:444-50.

2. Odell DD, Peleg K, Givon A, et al.; Israeli Trauma Group. Sternal fracture: isolated lesion versus polytrauma from associated extrasternal injuries - analysis of 1,867 cases. J Trauma Acute Care Surg 2013;75:448-52.

3. Khoriati AA, Rajakulasingam R, Shah R. Sternal fractures and their management. J Emerg Trauma Shock 2013;6:113-6.

\section{Competing interests: None declared.}

This article has been peer reviewed.

The authors have obtained patient consent.

Affiliations: Departments of Radiology (Sade) and Thoracic Surgery (Aydin), Ataturk University, Medical Faculty, Erzurum, Turkey

Correspondence to: Yener Aydin, dryeneraydin@hotmail.com

Clinical images are chosen because they are particularly intriguing, classic or dramatic. Submissions of clear, appropriately labelled high-resolution images must be accompanied by a figure caption. A brief explanation (300 words maximum) of the educational importance of the images with minimal references is required. The patient's written consent for publication must be obtained before submission. 